\title{
Herrnstein's equation for the rates of responding during concurrent schedules
}

\author{
FRANCES K. McSWEENEY, CAM L. MELVILLE, and J. E. WHIPPLE \\ Washington State University, Pullman, Washington
}

\begin{abstract}
A modification of the nonlinear curve-fitting procedure proposed by Wetherington and Lucas (1980) was used to assess how well Herrnstein's (1970) equation for the rates of responding during concurrent schedules described performance. The equation fitted some results very well, accounting for $80 \%$ or more of the variance in the data in studies that used moderateduration changeover delays and provided the same positive reinforcers, operanda, and simple schedules in the two components. The equation fitted the data poorly in other studies. The k parameter changed with several variables; it was not as constant as Herrnstein (1974) suggested. $R_{0}$ did not fit Herrnstein's interpretation as reinforcement from unprogrammed sources. Forty percent of all values of $R_{0}$ were negative, and another $23 \%$ were unreasonably large (greater than 50 reinforcers/h). The data suggest that Herrnstein's equation is not a general theory of concurrent-schedule responding, and that Herrnstein's interpretation of $\mathbf{k}$ and $\mathbf{R}_{\mathbf{0}}$ should be modified.
\end{abstract}

Herrnstein (1970) proposed that Equation 1 describes the relative rates of responding emitted by subjects responding on concurrent schedules:

$$
\frac{P_{1}}{P_{1}+P_{2}}=\frac{R_{1}}{R_{1}+R_{2}}
$$

He further proposed that Equation 2 describes the absolute rate of responding emitted during one component of a two-component concurrent schedule:

$$
P_{1}=\frac{k R_{1}}{R_{1}+R_{2}+R_{0}}
$$

Finally, he proposed that Equation 3 describes the rate of responding emitted during a simple schedule:

$$
P_{1}=\frac{k R_{1}}{R_{1}+R_{0}}
$$

In all three equations, $P_{1}$ is the rate of responding emitted for a rate of reinforcement equal to $R_{1}$. $P_{2}$ and $R_{2}$ are the rate of responding emitted during and the rate of reinforcement obtained from the other component of a concurrent schedule; $k$ and $\mathbf{R}_{0}$ are parameters estimated from the data (see McDowell, 1981). According to Herrnstein, k represents the subject's asymptotic rate of responding, which is constant if it is measured in constant response units (Herrnstein, 1974). $R_{0}$ represents the rate of unprogrammed reinforcers, measured in

Reprints may be obtained from Frances K. McSweeney, Department of Psychology, Washington State University, Pullman, Washington 99164. units equivalent to $R_{1}$ and $R_{2}$ (Herrnstein, 1970). It should remain constant if factors such as the subject's deprivation of the programmed reinforcer remain constant (Herrnstein \& Loveland, 1974).

Equations 1 and 3 have been studied extensively (e.g., de Villiers, 1977; de Villiers \& Herrnstein, 1976); they describe the data well when a few qualifications are added to Equation 1 (e.g., Baum, 1974; Myers \& Myers, 1977). But, Equation 2 and Herrnstein's interpretations of his parameters have been neglected. Some results appear to support, and others to contradict, Herrnstein's theory.

Several studies support Equation 2. First, the equation accounts for a large proportion of the variance in the rates of responding emitted during the components of concurrent schedules when subjects' body weights vary (McSweeney, 1975a). Second, the equation has had some success in predicting the rates of responding emitted during concurrent schedules from subjects' simple-schedule performance (McSweeney, 1978, 1982). Third, as predicted by the equation, the rate of responding emitted during one component of a concurrent schedule varies inversely with the rate of reinforcement obtained from the other component, regardless of whether those reinforcers are response dependent (e.g., Catania, 1963; Herrnstein, 1961) or response independent (Rachlin \& Baum, 1972). Finally, as predicted by the equation, the sum of the rates of responding may increase with increases in the sum of the rates of reinforcement that the components provide (e.g., Catania, 1963; Findley, 1958; McSweeney, 1977; Schmitt, 1974).

Other studies, however, have questioned both the basic form of Equation 2 and Herrnstein's interpretation of the $\mathbf{k}$ and $\mathbf{R}_{\mathbf{0}}$ parameters. First, according 
to Equations 2 and 3, the rate of responding emitted during a component of a concurrent schedule should be less than the rate of responding emitted during that same component appearing as a simple schedule. But, this may not be true (McSweeney \& Dericco, 1976). Second, some studies have failed to find the predicted increases in the sum of the rates of responding with increases in the sum of the rates of reinforcement when subjects respond on twocomponent (e.g., Fantino, Squires, Delbruck, \& Peterson, 1972; McSweeney, 1975a, 1975b) or threecomponent (e.g., Davison \& Hunter, 1976) concurrent schedules. Third, the rate of responding during one component of a concurrent schedule may vary with the rate of responding in the other component, not just with the alternative rate of reinforcement, as predicted by Equation 2 (Duncan \& Silberberg, 1982). Fourth, Herrnstein's parameters have not always behaved as his theory would predict when subjects respond on simple (e.g., Bradshaw, Szabadi, \& Bevan, 1978; de Villiers, 1977; de Villiers \& Herrnstein, 1976) or concurrent schedules (McSweeney, 1975a). Finally, Equation 2 predicts that the relative rates of responding during concurrent schedules will equal the relative rates of reinforcement that the components provide. Recent reviews of the literature show that the relative rates of responding undermatch, rather than exactly equal, the relative rates of reinforcement (e.g., Baum, 1979; Wearden \& Burgess, 1982).

These conflicting data have led others to formulate theories to deal with some of the violations of Equation 2 and of Herrnstein's interpretations of his parameters (e.g., McDowell, 1980; Staddon, in press). But Equation 2 deserves further study. Very few studies have carefully examined it; if true, Equation 2 would have important implications. Equation 2 and Equation 3, along with other aspects of Herrnstein's theory, imply that the absolute rates of responding emitted during simple, concurrent, and multiple schedules follow the same basic rules. Response rates are governed by the rate of reinforcement that they produce relative to all of the other rates of reinforcement available in the situation. If this implication of Herrnstein's theory is correct, then a general theory of responding on several schedules of reinforcement can be formulated, and theories that attribute responding on different schedules to fundamentally different processes must be incorrect (e.g., Rachlin, 1973). The present paper systematically evaluates Equation 2 by fitting it to the available data on concurrent-schedule responding.

\section{TREATMENT OF DATA}

The literature was searched for studies that adequately tested Herrnstein's equation, that is, studies that allowed accurate calculation of the absolute rates of responding and reinforcement during at least four concurrent schedules and that varied the rate of reinforcement over a wide range. Studies were included if they used standard concurrentschedule procedures, presented only two-component schedules, and conducted several sessions per schedule. Studies were not included if they altered response rates by reinforcing interresponse times, by presenting free reinforcers, or by signaling the availability of reinforcers.

Occasionally, a study that did not provide a perfect test of the theory but that provided unique data was used. For example, the data from Tustin \& Davison (1979) were read from a graph. Therefore, the rates of responding and reinforcement were not entirely clear. But this study provided the only information about a concurrent schedule in which a fixed-interval (FI) schedule followed changes from one component to the other. Studies that were included even though they violated one of these criteria have been indicated by footnotes when the results are presented.

A curve-fitting technique was applied to the results of these studies to assess how well Equation 2 fit and to calculate the best-fitting $k$ and $\mathbf{R}_{0}$ parameters. The technique used was a modification for concurrent schedules of the nonlinear, least squares method proposed by Wetherington and Lucas (1980) for simple schedules." Overdetermined estimates of $k$ and $R_{0}$ were obtained from the rates of responding and reinforcement during the components, by minimizing Equation 4 with respect to $k$ and $R_{0}$ :

$$
\sum_{i=1}^{n}\left[P_{i}-\left(\frac{k R_{1 i}}{R_{1 i}+R_{2 i}+\mathbf{R}_{0}}\right)\right]^{2} \text {. }
$$

This yielded Equation 5:

$$
\begin{aligned}
& \sum_{i=1}^{n} \frac{\mathbf{P}_{\mathrm{i}} \mathbf{R}_{1 \mathrm{i}}}{\left(\mathbf{R}_{1 \mathrm{i}}+\mathbf{R}_{2 \mathrm{i}}+\mathbf{R}_{\mathbf{0}}\right)} \sum_{\mathrm{i}=1}^{\mathrm{n}} \frac{\mathbf{R}_{1 \mathrm{i}}{ }^{2}}{\left(\mathbf{R}_{1 \mathrm{i}}+\mathbf{R}_{2 \mathrm{i}}+\mathbf{R}_{\mathbf{0}}\right)^{3}} \\
& =\sum_{\mathrm{i}=1}^{\mathrm{n}} \frac{\mathbf{P}_{\mathrm{i}} \mathbf{R}_{1 \mathrm{i}}}{\left(\mathbf{R}_{1 \mathrm{i}}+\mathbf{R}_{2 \mathrm{i}}+\mathbf{R}_{\mathbf{0}}\right)^{2}} \sum_{\mathrm{i}=1}^{\mathrm{n}} \frac{\mathbf{R}_{1 \mathrm{i}}{ }^{2}}{\left(\mathbf{R}_{1 \mathrm{i}}+\mathbf{R}_{2 \mathrm{i}}+\mathbf{R}_{\mathbf{0}}\right)^{2}}
\end{aligned}
$$

Equation 5 was solved for $\mathbf{R}_{\mathbf{0}}$. A solution was considered acceptable when the two sides of Equation 5 differed by no more than $\mathbf{. 0 0 0 0 1}$. The $k$ parameter was estimated from Equation 6 , using the value of $\mathbf{R}_{\mathbf{b}}$ obtained from Equation 5:

$$
k=\sum_{i=1}^{n} \frac{\mathbf{P}_{i} \mathbf{R}_{1 i}}{\left(\mathbf{R}_{1 i}+\mathbf{R}_{2 i}+\mathbf{R}_{0}\right)} \div \sum_{\mathrm{i}=1}^{n} \frac{\mathbf{R}_{1 i}{ }^{2}}{\left(\mathbf{R}_{1 i}+\mathbf{R}_{2 \mathrm{i}}+\mathbf{R}_{\mathbf{0}}\right)^{2}} .
$$

Generally, the author's divisions of the data were used in this analysis. For example, if more than one 
experiment was conducted, then the results of the different experiments were treated separately, rather than being pooled. Occasionally, however, data were separated into categories that were not used by the authors of the study. For example, Lobb and Davison (1975) and Trevett, Davison, and Williams (1972) conducted both concurrent variableinterval variable-interval (conc VI VI) and concurrent variable-interval fixed-interval (conc VI FI) schedules. The results for these different schedules were treated separately because doing so made the theory fit better.

\section{RESULTS}

Table 1 presents the size of the best-fitting $k$ and $R_{0}$ parameters, as well as the proportion of the variance in the data accounted for by Equation 2, for individual subjects and for the mean of all subjects. All data were analyzed by converting response rates to responses per minute and reinforcement rates to reinforcers per hour. Therefore, $k$ is reported in responses per minute and $\mathbf{R}_{\mathbf{o}}$ in reinforcers per hour.

In some cases, the error sum of squares in prediction using Equation 2 was greater than the sum of squares of the raw scores. Those cases have been indicated in Table 1 by the term "worse than random." In other cases, the values of $k$ and $R_{0}$ needed to solve Equations 5 and 6 were so large that they reduced the effect of $R_{1}$ and $R_{2}$. As a result, all values of $k$ and $R_{0}$ larger than those solutions also represented true mathematical solutions. Such solutions have been replaced by blank spaces in Table 1 .

Table 1

Best-Fitting $k$ and $R_{0}$ Parameters and the Proportion of the Variance in the Data Accounted for by Equation 2 for Individual Subjects and for the Mean of All Subjects

\begin{tabular}{|c|c|c|c|c|c|c|c|c|c|c|c|c|}
\hline \multirow[b]{2}{*}{ Subject } & \multicolumn{2}{|c|}{$\mathrm{k}$} & \multicolumn{2}{|c|}{$R_{0}$} & \multicolumn{2}{|c|}{$\begin{array}{c}\text { Proportion of } \\
\text { Variance }\end{array}$} & \multicolumn{2}{|c|}{ k } & \multicolumn{2}{|c|}{$\mathrm{R}_{\mathrm{o}}$} & \multicolumn{2}{|c|}{$\begin{array}{c}\text { Proportion of } \\
\text { Variance }\end{array}$} \\
\hline & k & $\mathrm{SD}$ & $\mathrm{R}_{0}$ & $\mathrm{SD}$ & $r^{2}$ & SR & $\mathbf{k}$ & $\mathrm{SD}$ & $\mathrm{R}_{0}$ & SD & $\mathrm{r}^{2}$ & SR \\
\hline
\end{tabular}

STUDIES THAT HERRNSTEIN'S EQUATION DESCRIBED WELL

Bradshaw, Szabadi, Bevan, and Ruddle (1979)

Data for Unsignaled Reinforcement Only

$\begin{array}{ll}\text { A.D. } & 313.4 \\ \text { M.W. } & 274.5 \\ \text { S.W. } & 291.0 \\ \text { Mean } & 291.9\end{array}$

\section{7}

53.2

96.3

24.2

117

117
243

294

Mean

11.3

113.7

28.0

31.4

$\begin{array}{lr}1 & 135.0 \\ 2 & 52.9 \\ 3 & 43.2 \\ \text { Mean } & 72.1\end{array}$

14.4
2.7
3.7
2.9

$\begin{array}{ll}1 & 83.8 \\ 2 & 42.4 \\ 3 & 34.6 \\ \text { Mean } & 51.6\end{array}$

16.2

7.0

2.1

7.2
Button 1

$\begin{array}{lr}-8.9 & 26.7 \\ -7.2 * & 11.1 \\ 17.3 & 17.9 \\ -1.3 & 5.9\end{array}$

$\begin{array}{rrr}.98 & 12.7 & 157.8 \\ .99 & 6.0 & 140.1 \\ .99 & 5.7 & 284.2 \\ .99 & 2.9 & 179.3\end{array}$

Catania (1963)

Key 1
-7.6
110.0
69.6
36.6

10.7
85.9
20.8
23.5

$\begin{array}{rrr}.94 & 3.5 & 79.3 \\ .71 & 11.9 & 116.4 \\ .91 & 3.9 & 57.6 \\ .88 & 5.3 & 83.7\end{array}$

Farley (1980) Experiment 1 Reinforcement Only

Left Key

$\begin{array}{lrrrr}21.6 & 8.5 & 1.00 & 1.6 & 208.0 \\ -7.4^{*} & 3.3 & 1.00 & .5 & 69.9 \\ -5.0^{*} & 5.4 & .98 & 1.5 & 58.4 \\ 2.9 & 3.0 & .99 & .6 & 99.0\end{array}$

Reinforcement and Punishment

\section{Left Key}

$\begin{array}{cr}-1.2 & 24.2 \\ -12.6 & 13.3 \\ -7.4^{*} & 3.3 \\ -7.7 & 12.7\end{array}$

$\begin{array}{rrr}.97 & 4.2 & 221.5 \\ .96 & 3.0 & 166.5 \\ .99 & .4 & 36.5 \\ .98 & 2.3 & 121.4\end{array}$

Leigland (1979)

Data From Baseline Conditions Only

Schedule 1

$\begin{array}{lrrrrrrr}\text { Group 1 } & 62.4 & 5.8 & 15.6 & 7.4 & .98 & 1.5 & 42.2 \\ \text { Group 2 } & 126.6 & 61.4 & 60.0 & 29.8 & .12 & 8.5 & 42.5 \\ \text { Mean } & 138.0 & 14.8 & 84.0 & 14.5 & .89 & 3.0 & 38.1\end{array}$

\section{(1)}

37.9
16.7
122.9
40.4

Button 2

$\begin{array}{rrrr}-34.0 & 184.4 & .97 & 14.2 \\ -17.4 & 76.3 & .99 & 2.9 \\ 30.2 & 439.5 & .72 & 39.7 \\ -14.9 & 194.9 & .98 & 10.3\end{array}$

Key 2

$\begin{array}{rrrrr}16.9 & 18.0 & 10.9 & .88 & 4.0 \\ 47.5 & 11.5 & 11.6 & .77 & 9.7 \\ 18.8 & 11.1 & 11.5 & .83 & 3.7 \\ 21.8 & 12.8 & 8.9 & .90 & 4.1\end{array}$

Right Key

$\begin{array}{rrrr}70.9 & 8.4 & 1.00 & 1.2 \\ 10.7 & 9.5 & .95 & 3.6 \\ 7.0 & 15.0 & .88 & 3.7 \\ 25.2 & 3.4 & .99 & 1.1\end{array}$

Right Key

$\begin{array}{crrr}95.0 & 119.4 & .96 & 5.9 \\ 177.0 & 69.9 & .97 & 2.6 \\ -9.0^{*} & 3.4 & .99 & .7 \\ 68.1 & 41.1 & .99 & 2.2\end{array}$

Schedule 2

$\begin{array}{rlrrr}3.2 & -13.1^{*} & 3.9 & .96 & 2.2 \\ 13.0 & -12.5 & 10.4 & .54 & 6.2 \\ 5.3 & -19.2^{*} & 5.5 & .90 & 3.1\end{array}$


Table 1 Continued

\begin{tabular}{|c|c|c|c|c|c|c|c|c|c|c|c|c|}
\hline \multirow[b]{2}{*}{ Subject } & \multicolumn{2}{|c|}{$\mathbf{k}$} & \multicolumn{2}{|c|}{$R_{0}$} & \multicolumn{2}{|c|}{$\begin{array}{l}\text { Proportion of } \\
\text { Variance }\end{array}$} & \multicolumn{2}{|c|}{$\mathrm{k}$} & \multicolumn{2}{|c|}{$\mathrm{R}_{0}$} & \multicolumn{2}{|c|}{$\begin{array}{c}\text { Proportion of } \\
\text { Variance }\end{array}$} \\
\hline & k & SD & $R_{0}$ & SD & $r^{2}$ & SR & $\mathbf{k}$ & SD & $R_{0}$ & SD & $r^{2}$ & SR \\
\hline
\end{tabular}

Lobb and Davison (1975)

Concurrent Fixed-Interval/Variable-Interval Schedule

\begin{tabular}{|c|c|c|c|c|c|c|c|c|c|c|c|c|}
\hline \multirow[b]{2}{*}{ Mean } & \multicolumn{6}{|c|}{ Fixed-Interval Component } & \multicolumn{6}{|c|}{ Variable-Interval Component } \\
\hline & 76.1 & 15.1 & 6.9 & 15.2 & .87 & 4.7 & 90.0 & 29.0 & -1.7 & 13.7 & .74 & 8.3 \\
\hline & \multicolumn{12}{|c|}{ Concurrent Variable-Interval/Variable-Interval Schedule } \\
\hline & \multicolumn{6}{|c|}{ Red Component } & \multicolumn{6}{|c|}{ Green Component } \\
\hline
\end{tabular}

McSweeney (1975b)

\section{Constant Component}

$\begin{array}{lrrrrrrr}8422 & 35.6 & 1.1 & 25.5 & 3.0 & .98 & .4 & 20.6 \\ 8772 & 39.7 & 5.0 & 4.9 & 15.5 & .92 & 1.9 & 22.3 \\ 8845 & 31.2 & 2.9 & 39.8 & 14.1 & .89 & .8 & 20.9 \\ 8895 & & & & & & & 21.4 \\ \text { Mean } & 25.5 & 2.8 & -2.1 & 11.0 & .93 & 1.2 & 20.9\end{array}$

Norman and McSweeney (1978)

Right Lever

$\begin{array}{lrrrrrrrrrrrr}1 & 41.3 & 3.6 & -1.6 & 13.9 & .58 & 2.7 & 89.7 & 13.3 & 61.0 & 36.2 & .90 & 2.3 \\ 2 & & & & & & & & 60.0 & 4.2 & 20.0 & 16.9 & .99 \\ 3 & 89.3 & 7.9 & 44.3 & 14.9 & .87 & 4.0 & 76.0 & 9.6 & 62.0 & 28.5 & .83 & 1.6 \\ 4 & 68.3 & 4.8 & 73.3 & 11.3 & .97 & 1.5 & 73.0 & 4.7 & 70.0 & 15.9 & .98 & .6 \\ 5 & 132.9 & 15.7 & 184.1 & 43.4 & .98 & 2.9 & 41.0 & 25.1 & -25.6 & 60.3 & .99 & 1.0 \\ \text { Mean } & 136.2 & 4.7 & 178.5 & 10.0 & .99 & 1.2 & 64.2 & 4.7 & 27.5 & 17.5 & .99 & .6\end{array}$

Tustin and Davison (1979) ${ }^{1}$

Fixed-Interval 0-Sec Changeover Delay

\begin{tabular}{|c|c|c|c|c|c|c|c|c|c|c|c|c|}
\hline & \multicolumn{6}{|c|}{ Key 1} & \multicolumn{6}{|c|}{ Key 2} \\
\hline 22 & 73.5 & 29.1 & 19.1 & 35.6 & .99 & 9.4 & 122.1 & 11.9 & 64.0 & 20.0 & .90 & 4.3 \\
\hline 23 & 78.1 & 22.8 & 3.3 & 44.1 & .91 & 6.8 & 138.4 & 36.5 & 130.0 & 182.0 & .67 & 6.8 \\
\hline 24 & 69.8 & 27.1 & 48.0 & 33.6 & .91 & 4.8 & 99.2 & 16.4 & 75.0 & 43.0 & .80 & 4.3 \\
\hline 26 & & & & & & & 368 & 7.8 & 17.5 & 44.9 & .80 & 1 \\
\hline Mean & 78.8 & 13.2 & 3.9 & 19.1 & .99 & 1.5 & 120.6 & 6.2 & 71.5 & 8.2 & .96 & 4 \\
\hline
\end{tabular}

\section{STUDIES THAT HERRNSTEIN'S EQUATION DESCRIBED TO SOME EXTENT}

Bourland and Miller $(1981)^{2}$

Red Component

333

$\begin{array}{lr}161 & 12.9 \\ 163 & 7.4 \\ 164 & 168.9 \\ 165 & 12.2 \\ 166 & 7.6 \\ \text { Mean } & 24.9\end{array}$

Median
$119.0 \quad 380.4$

$\begin{array}{llr}5.1 & -18.3 & 20.8\end{array}$ $\begin{array}{rr}.24 & 27.4 \\ .98 & 1.3\end{array}$

worse than random

Green Component

$\begin{array}{llrrrr}40.1 & 6.1 & -8.3 & 9.6 & .96 & 3.7 \\ 20.7 & 6.1 & -89.9 & 22.0 & 98^{3} & 1.3\end{array}$

Davison and Ferguson (1978)

Lever

$\begin{array}{rcr}1.5 & -69.2^{*} & 7.9 \\ .4 & -86.2^{*} & 2.1 \\ 51.2 & 178.0 & 66.6 \\ 2.7 & -50.9 & 23.1 \\ 1.2 & -8.7^{*} & 11.5 \\ 3.0 & -24.6^{*} & 9.6\end{array}$

Graft, Lea, and Whitworth (1977)

Chamber 2

$\begin{array}{rrr}.93 & 1.8 & \\ .99 & .3 & \\ .79 & 6.1 & 67.8 \\ .90 & 1.7 & 326.0 \\ .97 & .3 & 112.9 \\ .97 & 1.0 & \end{array}$

$\begin{array}{llllll}26.0 & 11.5 & .68 & .3 & 3.3\end{array}$

Chamber 5

$$
\text { Key }
$$

$\begin{array}{lcccr}21.8 & -49.4^{*} & 22.5 & .60 & 9.8 \\ 81.4 & 166.0 & 59.9 & .48 & 13.7 \\ 47.4 & -4.8 & 22.2 & .01 & 14.9\end{array}$

1.4

3.2

4.0
1.5 
Table 1 Continued

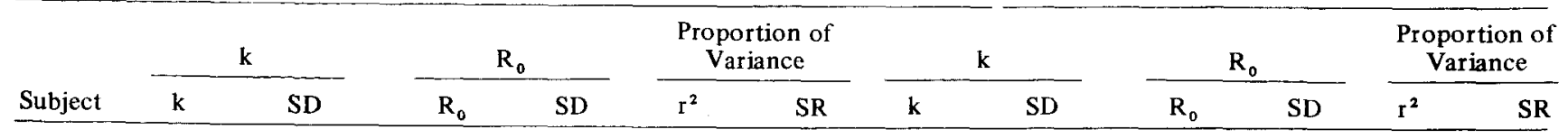

La Bounty and Reynolds (1973) ${ }^{2}$

Fixed-Interval Component

\begin{tabular}{|c|c|c|c|c|c|c|c|c|c|c|c|c|}
\hline & \multicolumn{6}{|c|}{ Fixed-Interval Component } & \multicolumn{6}{|c|}{ Fixed-Ratio Component } \\
\hline 867 & 602.0 & 116.0 & 87.1 & 28.3 & .66 & 15.8 & 180.0 & 77.0 & 26.0 & 14.6 & .98 & 10.5 \\
\hline 464 & 450.7 & 57.2 & 52.6 & 12.2 & .93 & 6.3 & 155.0 & 25.9 & 2.8 & 9.8 & .90 & 15.2 \\
\hline 875 & 649.7 & 138.5 & 115.0 & 46.8 & .49 & 13.8 & 251.0 & 89.0 & 20.9 & 24.3 & .83 & 26.3 \\
\hline 877 & & & & & & & 316.5 & 528.8 & 174.1 & 101.1 & .90 & 23.7 \\
\hline 866 & 98.6 & 74.7 & 7.2 & 23.8 & .12 & 9.40 & & & & & & \\
\hline
\end{tabular}

Matthews and Temple (1979)

Concurrent Hay-Meal Schedules Only

Hay Component

\begin{tabular}{|c|c|c|c|c|c|c|c|c|c|c|c|c|}
\hline & \multicolumn{6}{|c|}{ Hay Component } & \multicolumn{6}{|c|}{ Meal Component } \\
\hline 963 & 4.1 & 22.3 & 39.6 & 83.4 & .40 & 4.8 & worse & indom & & & & \\
\hline 964 & 4.7 & 5.5 & -13.9 & 26.2 & .02 & 1.1 & worse & andom & & & & \\
\hline 965 & 12.0 & 16.1 & $-27.0^{*}$ & 23.8 & .17 & 3.4 & 12.4 & 18.7 & $-24.5^{*}$ & 24.2 & .12 & 3.6 \\
\hline 968 & 5.7 & 2.9 & -3.6 & 20.2 & .96 & .3 & 7.6 & 5.8 & -6.3 & 23.9 & .45 & 1.2 \\
\hline Mean & 4.7 & 6.0 & $-31.1 *$ & 19.8 & .47 & 1.3 & 7.9 & 7.7 & $-15.0^{*}$ & 22.1 & .17 & 1.6 \\
\hline
\end{tabular}

McSweeney (1975a)

$80 \%$ Free-Feeding Body Weights ${ }^{2}$

Red Component

\begin{tabular}{lrrrrrrr}
394 & 69.7 & 9.6 & -1.6 & 92.4 & .97 & 6.1 & 87.6 \\
259 & 71.9 & 22.5 & 29.6 & 21.1 & .85 & 4.1 & \\
5 & 50.6 & 33.6 & -9.6 & 21.4 & .12 & 9.1 & 92.3 \\
& & & \multicolumn{7}{c}{$105 \%$} & Free-Feeding Body Weight
\end{tabular}

Red Component

$\begin{array}{lrrrrrr}394 & 58.8 & 20.1 & 7.3 & 72.3 & .98 & 2.4 \\ 259 & 31.0 & 2.0 & -9.2 & 3.1 & .97 & 2.0 \\ 5 & 49.9 & 4.4 & 2.2 & 4.1 & .95 & 3.4 \\ \text { Mean } & 97.7 & 35.6 & 26.2 & 69.1 & 1.00 & 15.7\end{array}$

McSweeney (1977)

More Favorable Component

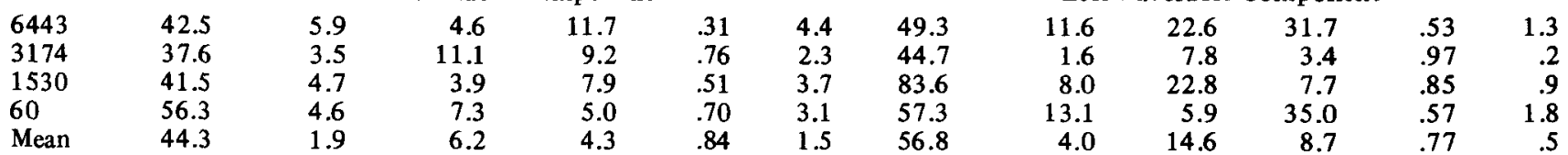

McSweeney (1978)*

5-Sec Changeover Delay ${ }^{3}$

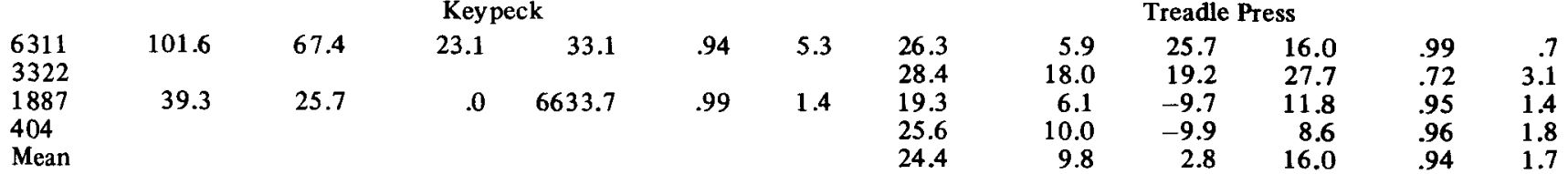

20-Sec Changeover Delay

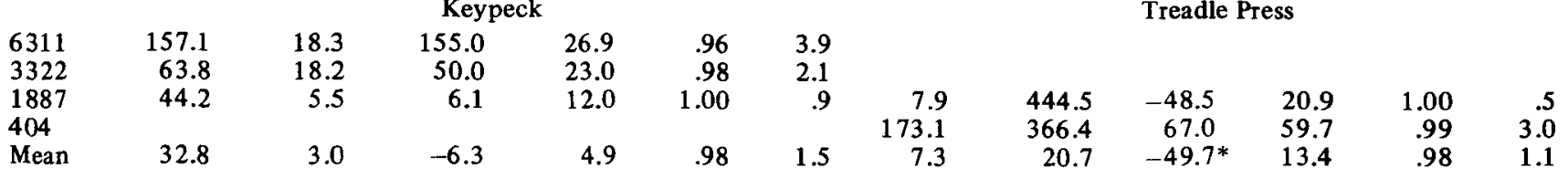


Table 1 Continued

\begin{tabular}{|c|c|c|c|c|c|c|c|c|c|c|c|c|}
\hline \multirow[b]{2}{*}{ Subject } & \multicolumn{2}{|c|}{$\mathrm{k}$} & \multicolumn{2}{|c|}{$\mathbf{R}_{0}$} & \multicolumn{2}{|c|}{$\begin{array}{l}\text { Proportion of } \\
\text { Variance }\end{array}$} & \multicolumn{2}{|c|}{$\mathbf{k}$} & \multicolumn{2}{|c|}{$\mathbf{R}_{\mathbf{0}}$} & \multicolumn{2}{|c|}{$\begin{array}{c}\text { Proportion of } \\
\text { Variance }\end{array}$} \\
\hline & k & SD & $\mathbf{R}_{0}$ & SD & $\mathrm{r}^{2}$ & SR & k & SD & $\mathbf{R}_{\mathbf{0}}$ & SD & $r^{2}$ & SR \\
\hline
\end{tabular}

\section{Rider (1979) ${ }^{2}$}

Fixed-Ratio Component

\begin{tabular}{|c|c|c|c|c|c|c|c|}
\hline & & & & & & & \\
\hline 25 & 134.8 & 115.5 & 75.0 & 115.4 & .88 & 8.5 & \\
\hline 26 & 152.8 & 34.6 & 140.0 & 35.8 & .99 & 1.9 & 88.0 \\
\hline 33 & 85.5 & 20.0 & 25.1 & 48.1 & .98 & 2.7 & 68.1 \\
\hline 35 & 184.2 & 77.7 & $-29.2^{*}$ & 80.6 & .98 & 9.5 & 147.1 \\
\hline 40 & & & & & & & \\
\hline 41 & & & & & & & \\
\hline 46 & 82.7 & 66.2 & $-36.3^{*}$ & 62.2 & .99 & 2.8 & 189.5 \\
\hline & & & & & Ride & 1981) & \\
\hline & & & -Interval & mponen & & & \\
\hline $\begin{array}{l}102 \\
103\end{array}$ & & & & & & & $\begin{array}{l}111.5 \\
118.8\end{array}$ \\
\hline 104 & 83.0 & 10.8 & 60.7 & 20.8 & .95 & 2.7 & 135.8 \\
\hline 105 & 41.2 & 5.8 & 6.0 & 11.5 & .97 & 1.7 & 175.0 \\
\hline 106 & & & & & & & 135.7 \\
\hline
\end{tabular}

Variable- or Mixed-Ratio Component

$\begin{array}{rcrrr}10.0 & 33.0 & 34.9 & .99 & 2.0 \\ 8.1 & 16.5 & 16.8 & .97 & 2.7 \\ 57.2 & -82.6^{*} & 72.0 & .93 & 18.8\end{array}$

$\begin{array}{llll}66.2 & 68.9 & 74.3 & .75\end{array}$

23.2

Variable-Ratio Component

$\begin{array}{rrrrr}62.9 & -10.0 & 30.1 & .47 & 24.8 \\ 972.0 & 9.8 & 32.1 & .65 & 17.0 \\ 94.8 & 8.8 & 30.4 & .61 & 16.3 \\ 107.0 & 11.2 & 26.4 & .66 & 19.2 \\ 5006.2 & 63.2 & 73.2 & .76 & 12.7\end{array}$

Wheatley and Engberg (1978)

Concurrent Keypeck Treadle-Press Schedules

KT1
KT2
KT3
Mean

Mean

$\begin{array}{lr}21 & 169.2 \\ 22 & 92.6 \\ 23 & 213.2 \\ 24 & 91.6 \\ 25 & 187.0 \\ 26 & 193.6 \\ \text { Mean } & 152.6\end{array}$

Keypeck Component

$\begin{array}{rr}44.7 & 3404.3 \\ 62.0 & 3808.2 \\ 39.3 & 355.5\end{array}$

Treadle-Press Component

$\begin{array}{crrrrrrrrr}-41.1^{*} & 24.6 & .87 & 11.0 & 32.8 & 2196.0 & 50.0 & 80.5 & .75 & 3.8 \\ 7.3 & 36.2 & .81 & 6.1 & & & & & & \\ -42.3^{*} & 25.4 & .64 & 11.1 & 47.6 & 1613.5 & 68.0 & 70.0 & .90 & 2.5\end{array}$

White and Davison (1973)

Red Component

\begin{tabular}{rrrrrr}
\multicolumn{7}{c}{ Red Component } \\
103.9 & 102.8 & 127.1 & .66 & 23.6 & 141.7 \\
35.0 & 11.5 & 51.6 & .85 & 8.3 & 94.0 \\
117.2 & 153.4 & 114.5 & .80 & 19.9 & 146.0 \\
32.5 & 25.8 & 97.4 & .66 & 14.5 & 80.5 \\
78.0 & 66.7 & 142.0 & .71 & 27.0 & 117.9 \\
98.1 & 51.4 & 93.5 & .63 & 29.5 & 178.7 \\
61.6 & 57.3 & 73.3 & .78 & 16.9 & 129.5
\end{tabular}

\begin{tabular}{rrrrr}
\multicolumn{5}{c}{ Green Component } \\
49.4 & 10.3 & 87.6 & .90 & 9.5 \\
54.3 & 8.6 & 115.3 & .71 & 9.8 \\
79.1 & 12.9 & 114.4 & .62 & 14.8 \\
39.6 & 2.4 & 107.4 & .85 & 6.2 \\
58.7 & 4.2 & 94.2 & .58 & 13.1 \\
123.1 & 33.3 & 126.6 & .63 & 22.4 \\
59.2 & 7.4 & 99.1 & .81 & 10.5
\end{tabular}

\section{STUDIES THAT HERRNSTEIN'S EQUATION DESCRIBED POORLY}

Herrnstein and Heyman (1979)

\begin{tabular}{|c|c|c|c|c|}
\hline $\begin{array}{l}3 \\
83\end{array}$ & $\begin{array}{cc}68.4 & 12.6 \\
\text { worse than random }\end{array}$ & -3.7 & 30.8 & .47 \\
\hline $\begin{array}{l}365 \\
473\end{array}$ & $\begin{array}{ll}137.0 & 6.0 \\
\text { worse than random }\end{array}$ & 90.9 & 17.7 & .99 \\
\hline
\end{tabular}

Variable-Ratio Component

Hollard and Davison (1971)

\begin{tabular}{lrrrrrrr}
93 & 71.2 & 117.2 & $-11.7^{*}$ & 20.1 & .76 & 12.1 & 51.5 \\
95 & & & & & & & \\
119 & & & & & & & \\
Mean & 47.6 & 20.1 & -27.9 & 14.7 & .54 & 20.7 & 67.3 \\
\multicolumn{7}{c}{ Left Lever } & Logue and de Villiers (1978)
\end{tabular}

R7

Left Lever

$\begin{array}{llllll}250.0 & 61.3 & 166.0 & 90.6 & .73 & 15.9\end{array}$

Brain Stimulation Component

$\begin{array}{lllll}31.4 & 53.0 & 74.2 & .60 & 6.9\end{array}$

R9

Mean 
Table 1 Continued

\begin{tabular}{|c|c|c|c|c|c|c|c|c|c|c|c|c|}
\hline \multirow[b]{2}{*}{ Subject } & \multicolumn{2}{|c|}{ k } & \multicolumn{2}{|c|}{$\mathbf{R}_{\mathbf{0}}$} & \multicolumn{2}{|c|}{$\begin{array}{c}\text { Proportion of } \\
\text { Variance }\end{array}$} & \multicolumn{2}{|c|}{$\mathrm{k}$} & \multicolumn{2}{|c|}{$\mathbf{R}_{0}$} & \multicolumn{2}{|c|}{$\begin{array}{l}\text { Proportion of } \\
\text { Variance }\end{array}$} \\
\hline & k & SD & $\mathbf{R}_{\mathbf{0}}$ & $\mathrm{SD}$ & $r^{2}$ & $\mathrm{SR}$ & k & SD & $R_{0}$ & $\mathrm{SD}$ & $r^{2}$ & SR \\
\hline
\end{tabular}

\begin{tabular}{|c|c|c|c|c|c|c|c|c|c|c|c|c|}
\hline \multirow[b]{3}{*}{$\begin{array}{l}6311 \\
3322 \\
1887 \\
404 \\
\text { Mean }\end{array}$} & \multicolumn{11}{|c|}{$\begin{array}{c}\text { McSweeney (1978) } \\
\text { 0-Sec Changeover Delay }\end{array}$} & \\
\hline & \multicolumn{6}{|c|}{ Keypeck Component } & \multicolumn{6}{|c|}{ Treadle-Press Component } \\
\hline & $\begin{array}{l}50.6 \\
34.9 \\
70.0 \\
74.5 \\
55.6\end{array}$ & $\begin{array}{l}32.6 \\
16.6 \\
52.6 \\
53.8 \\
35.7\end{array}$ & $\begin{array}{r}-3.7 \\
25.4 \\
107.0 \\
62.5 \\
35.7\end{array}$ & $\begin{array}{l}22.4 \\
26.3 \\
61.2 \\
45.2 \\
34.5\end{array}$ & $\begin{array}{l}.59 \\
.88 \\
.86 \\
.81 \\
.77\end{array}$ & $\begin{array}{l}7.0 \\
2.5 \\
4.7 \\
5.9 \\
5.0\end{array}$ & $\begin{array}{c}22.8 \\
\text { worse } t \\
\text { worse } t \\
54.3 \\
\text { worse } t\end{array}$ & $\begin{array}{c}62.4 \\
\text { an random } \\
\text { an random } \\
46.9 \\
\text { an random }\end{array}$ & 20.0 & 49.1 & .07 & 4.2 \\
\hline \multicolumn{13}{|c|}{ Nevin (1971) } \\
\hline & \multicolumn{6}{|c|}{ Fixed-Interval Schedule } & \multicolumn{6}{|c|}{ Variable-Interval Schedule } \\
\hline $\begin{array}{l}58 \\
59 \\
60 \\
\text { Mean }\end{array}$ & & & & & & & $\begin{array}{r}124.6 \\
95.6 \\
84.1 \\
100.6\end{array}$ & $\begin{array}{r}12522.6 \\
8765.6 \\
8004.0 \\
9404.6\end{array}$ & $\begin{array}{r}9.8 \\
12.6 \\
18.8 \\
12.4\end{array}$ & $\begin{array}{l}31.1 \\
34.8 \\
35.5 \\
32.5\end{array}$ & $\begin{array}{l}.83 \\
.72 \\
.85 \\
.85\end{array}$ & $\begin{array}{l}7.9 \\
8.3 \\
4.7 \\
5.9\end{array}$ \\
\hline
\end{tabular}

Pliskoff, Cicerone, and Nelson (1978) Experiment $1^{2}$

First Replication

Red Key Green Key

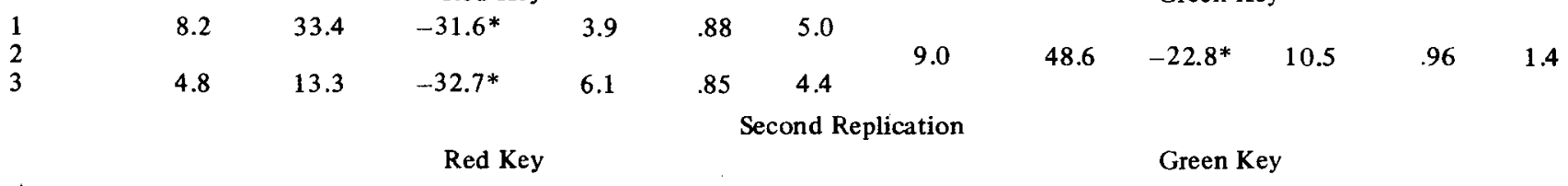

Schmitt (1974) Experiment 1

\begin{tabular}{ll} 
S & \\
Gi & 42.0 \\
J & 47.4 \\
Go & 46.7 \\
Mean & 36.3 \\
\hline
\end{tabular}

103

104

108

Mean

Red Key
More Favorable Component

Less Favorable Component

worse than random

$\begin{array}{rrrrr}36.7 & 11.4 & 29.9 & .75 & 20.1 \\ 9.0 & -9.3 & 19.2 & .29 & 8.4 \\ 81.4 & -180.2 & 181.1 & .81 & 17.1 \\ 3.7 & 23.9 & 40.4 & .86 & 2.4 \\ 4.4 & 28.8 & 11.1 & .96 & 2.0\end{array}$

worse than random

worse than random

$\begin{array}{llllll}143.0 & 55.6 & 37.5 & 71.0 & .31 & 7.7\end{array}$

Stubbs and Pliskoff (1969)

2-Sec Changeover Delay Data

Green Key

Trevett, Davison, and Williams (1972)

Concurrent Fixed-Interval/Variable Interval Schedules

worse than random

worse than random

$\begin{array}{rccrrr}71.6 & 31.1 & -38.2^{*} & 9.5 & .61 & 14.9 \\ 129.4 & 51.5 & 26.0 & 23.8 & .69 & 9.3\end{array}$ 
Table 1 Continued

\begin{tabular}{|c|c|c|c|c|c|c|c|c|c|c|c|c|}
\hline \multirow[b]{2}{*}{ Subject } & \multicolumn{2}{|c|}{$\mathbf{k}$} & \multicolumn{2}{|c|}{$R_{0}$} & \multicolumn{2}{|c|}{$\begin{array}{c}\begin{array}{c}\text { Proportion of } \\
\text { Variance }\end{array} \\
\end{array}$} & \multicolumn{2}{|c|}{$\mathbf{k}$} & \multicolumn{2}{|c|}{$R_{0}$} & \multicolumn{2}{|c|}{$\begin{array}{l}\text { Proportion of } \\
\quad \text { Variance }\end{array}$} \\
\hline & $k$ & SD & $R_{0}$ & SD & $r^{2}$ & SR & $\mathbf{k}$ & SD & $\mathrm{R}_{0}$ & SD & $r^{2}$ & SR \\
\hline
\end{tabular}

Concurrent Variable-Interval/Variable-Interval Schedule

$\begin{array}{lllllll}101 & & & & & & \\ 102 & 78.0 & 16.3 & -14.8 & 27.0 & .99 & 2.4 \\ 105 & & \end{array}$

106

Mean

Concurrent Variable-Interval/Variable-Interval Schedule
Component 1

Component 2

worse than random

$\begin{array}{llllll}60.5 & 24.5 & -35.6 & 15.3 & .96 & 11.2\end{array}$

Tustin and Davison (1979)

Fixed-Interval 180-Sec Changeover Delay Component 1

\begin{tabular}{|c|c|c|c|c|c|c|c|c|}
\hline \multicolumn{7}{|c|}{ Component 1} & & on \\
\hline 21 & 46.5 & 12.3 & $-40.2^{*}$ & 20.3 & .94 & 4.1 & & \\
\hline 22 & 15.5 & 49.3 & $-87.1 *$ & 47.7 & .01 & 10.8 & & \\
\hline 23 & 31.9 & 20.8 & -17.2 & 84.5 & .85 & 4.5 & 57.3 & 21.5 \\
\hline 24 & worse & $\mathrm{n}$ randon & & & & & & \\
\hline 25 & 50.2 & 53.0 & 9.5 & 39.7 & .88 & 5.1 & & \\
\hline 26 & 21.9 & 1028.8 & $-47.3^{*}$ & 17.9 & .92 & 2.3 & wo & n random \\
\hline Mean & 33.2 & 1555.3 & $-37.0^{*}$ & 15.6 & .94 & 2.9 & & \\
\hline
\end{tabular}

Component 2

Fixed-Interval 360-Sec Changeover Delay Component 1

\begin{tabular}{|c|c|c|c|c|c|c|c|}
\hline & & & 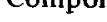 & & & & \\
\hline 21 & & & & & & & worse than random \\
\hline & 34.7 & 7.0 & $-40.0^{*}$ & 20.8 & .74 & 4.3 & worse than random \\
\hline 23 & 25.1 & 5.3 & -45.4 & 28.4 & .61 & 4.5 & worse than random \\
\hline $\begin{array}{l}24 \\
25\end{array}$ & 18.5 & 2.4 & $-56.1^{*}$ & 18.8 & .48 & 2.3 & $\begin{array}{l}\text { worse than random } \\
\text { worse than random }\end{array}$ \\
\hline $\begin{array}{l}26 \\
\text { Mean }\end{array}$ & 12.8 & 3.5 & $-53.7^{*}$ & 41.4 & .23 & 3.3 & $\begin{array}{l}\text { worse than random } \\
\text { worse than random }\end{array}$ \\
\hline
\end{tabular}

Component 2

Wheatley and Engberg (1978)

Concurrent Treadle-Press/Treadle-Press Schedules

\begin{tabular}{llllllll} 
TT1 & 44.5 & 2643.6 & 74.0 & 83.5 & .51 & 4.5 & worse than random \\
TT2 & 30.3 & 1135.4 & -2.2 & 57.9 & .73 & 5.4 & \\
Mean & 30.9 & 1481.8 & 9.3 & 49.7 & .73 & 4.2 & \\
\hline
\end{tabular}

Treadle 2

Note-SR = square root of the error variance. $\quad{ }^{*} R_{0}$ was significantly less than 0 by the binomial test $(p<.05) . \quad{ }^{1}$ These data were read from a figure and may not be entirely accurate. ${ }^{2}$ The mean was not calculated because different subjects responded on differ. ent schedules. ${ }^{3}$ These calculations are based on only three data points. "The raw data do not appear in this study, but they were obtained from McSweeney.

They have not been reported because they do not have meaningful implications for Herrnstein's theory.

The standard deviations of $k$ and $R_{0}$ and the square root of the error variance also appear in Table 1 (cf. Davison, 1982). Point estimates of $k$ were obtained by using the best-fitting $R_{0}$ reported in Table 1 to calculate a value of $\mathbf{k}$ for each individual schedule that entered the calculations for that $k$. The number reported after $k$ in the table is the standard deviation of these point estimates. Point estimates of $R_{0}$ were obtained by using the best-fitting $k$ reported in Table 1 to calculate a value of $R_{0}$ for each individual schedule that entered the calculations for that $R_{0}$. The standard deviation of these points appears in parentheses following $\mathbf{R}_{0}$ on the table. The square root of the error variance was calculated by using the best-fitting $k$ and $R_{0}$ to calculate a predicted response rate for each individual schedule. The square root of the error variance is the standard deviation of the difference between the obtained and predicted rates for each individual schedule that entered the calculations for that proportion of the variance. It appears in the columns immediately to the right of the columns containing proportion of the variance in Table 1. The large standard deviations that occasionally occurred (Hollard \& Davison, 1971; McSweeney, 1978; Nevin, 1971; Rider, 1981; Tustin \& Davison, 1979; Wheatley \& Engberg, 1978) are an artifact of extremely large 
point values of $k$ or $\mathbf{R}_{\mathbf{0}}$, produced by a rate of reinforcement or a rate of responding equal to zero for one or more schedules.

In order to determine whether the negative values of $\mathbf{R}_{\mathbf{0}}$ reported in Table 1 differed significantly from 0 , the binomial test (Siegel, 1956) was applied to their point estimates. The binomial test was used instead of a parametric test because the sampling distributions of the point estimates of $R_{0}$ were not normal. Negative $R_{0}$ values that were significantly less than $0(p<.05$, one-tailed) are followed by an asterisk in Table 1.

The studies presented in Table 1 have been divided into three categories: those that Herrnstein's equation described well, those that it described somewhat, and those that it described poorly. Herrnstein's equation described the data well in studies for which it accounted for $80 \%$ or more of the variance in the data on the average and for which "no solutions" or "worse than random solutions" were found in fewer than $10 \%$ of the cases. Herrnstein's equation described the data to some extent in studies for which it accounted for less than $80 \%$ of the variance on the average, and for which no solutions or worse-than-random solutions were found in several, but fewer than half, of the cases. Herrnstein's equation described the data poorly for studies in which it accounted for less than $80 \%$ of the variance in the data on the average and for which no solutions or worse-than-random solutions were found in half of the cases or more.
Table 2 summarizes the procedures used in the studies presented in Table 1 . The studies have been divided into the same three categories used in $\mathrm{Ta}$ ble 1. Most of the notation used in Table 2 is selfexplanatory. A two-operanda concurrent schedule provides the components of the concurrent schedule on two different operanda. The subject changes from one component to the other by moving from one operandum to the other. A Findley concurrent schedule provides the components of the concurrent schedule on a single operandum. The subject changes from one component to the other by responding on a second operandum. A changeover delay (COD) is a period of time following a change from one component to the other during which no reinforcers are presented. A changeover schedule (COS) is a schedule that must be completed following a change from one component to the other before any reinforcers may be obtained. The series used to schedule the interreinforcer intervals during VI and variable ratio (VR) schedules appear under the schedules on the table. The Catania and Reynolds series comes from Catania and Reynolds (1968, Appendix II), the Fleshler and Hoffman series from Fleshler and Hoffman (1962), and the Segal series from Segal (1964).

Several conclusions emerge from Tables 1 and 2. First, Herrnstein's equation described the data well in fewer than one-third of the studies. Only 8 studies fell into the category of a good fit, while 12 fell into the intermediate category and 11 fell into the poor category.

Table 2

Procedures Used by Studies Reported in Table 1

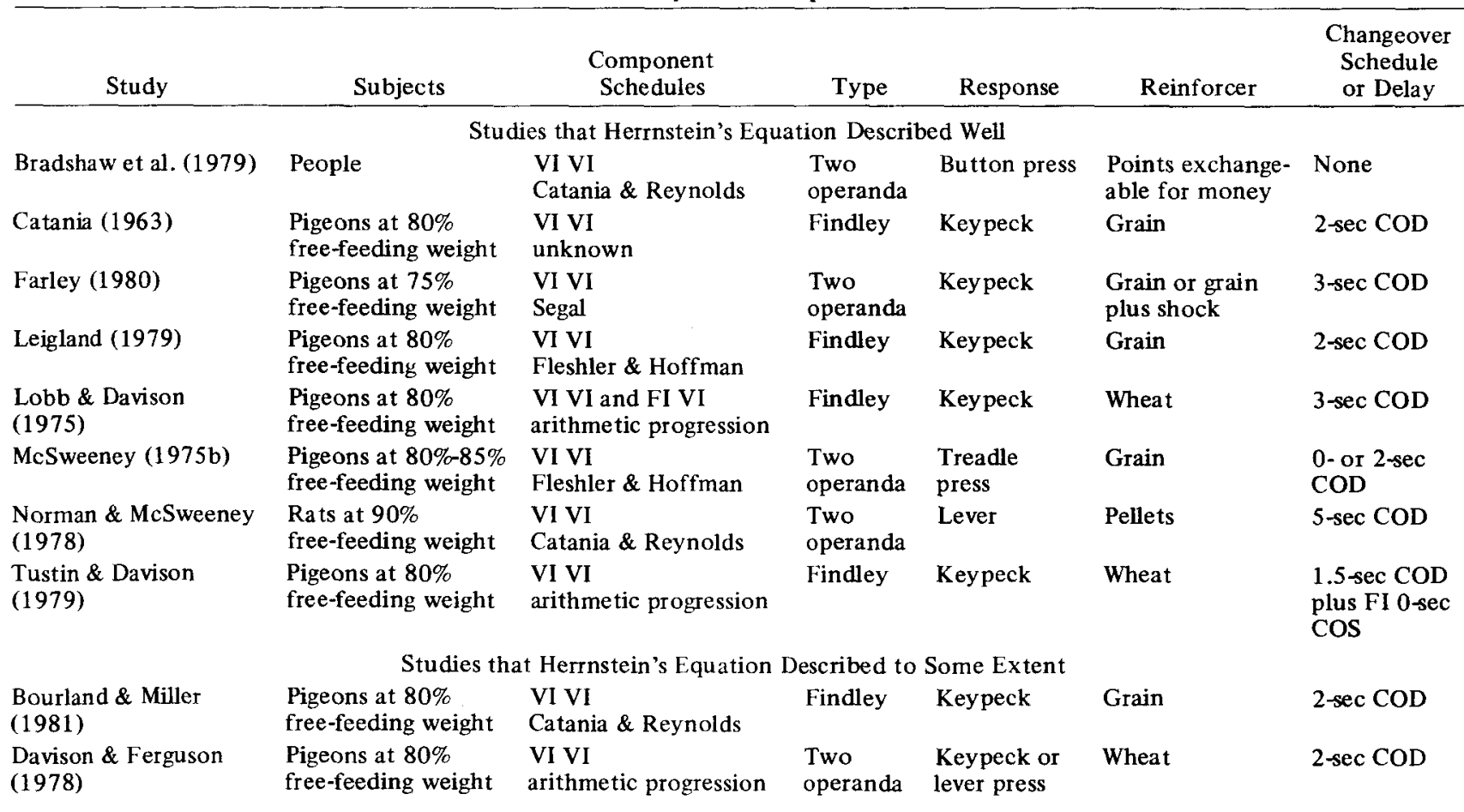


Table 2 Continued

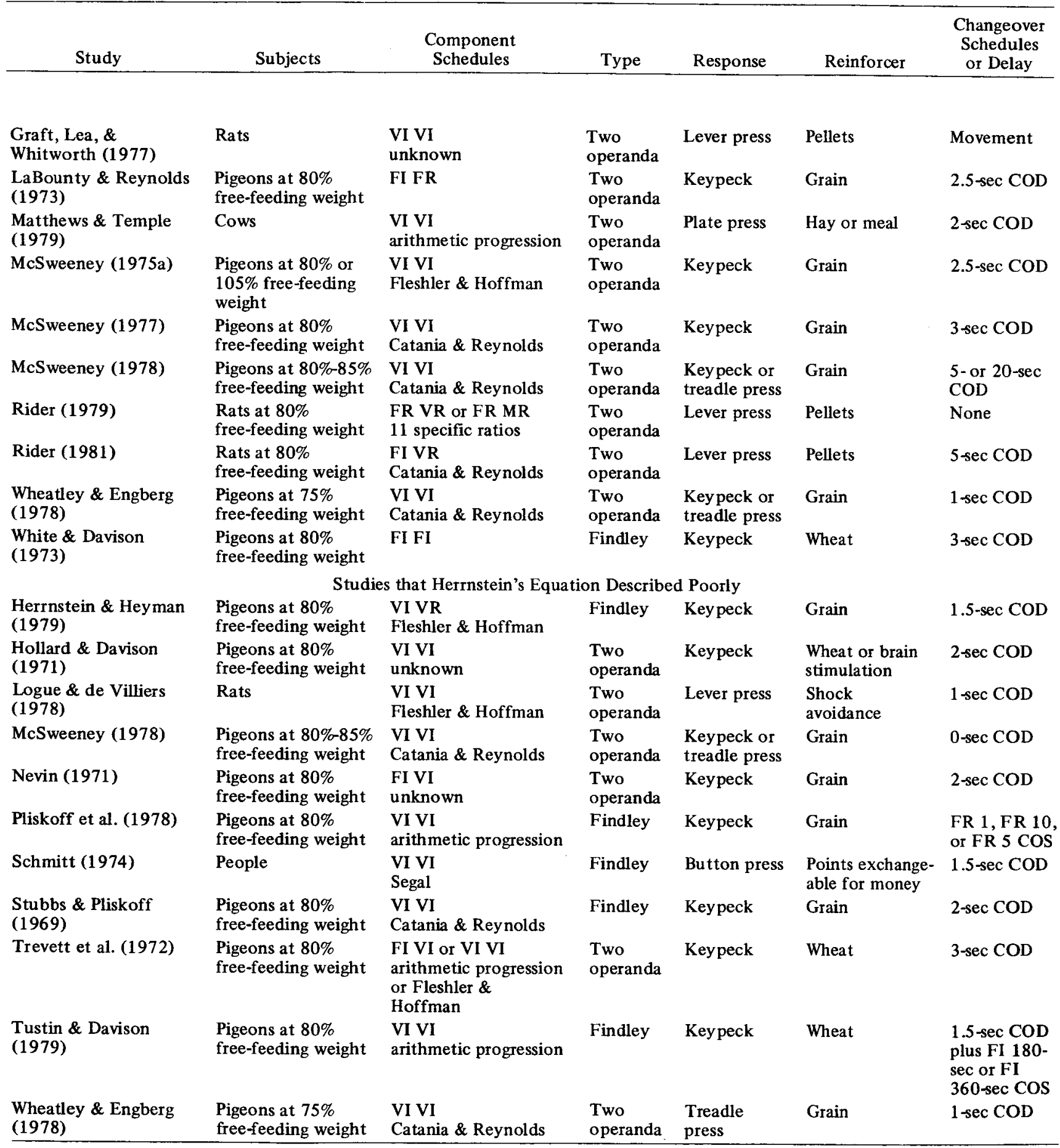

Several factors did not influence the category into which studies fell. Findley and two-operanda procedures appeared in all categories. Different methods of programming interreinforcer intervals fell into all categories. Different species and maintenance conditions of subjects, as well as some choices of reinforcers (e.g., grain, wheat, points ex- changeable for money) appeared in all categories. Different types of operanda appeared in all categories when the same type of operandum was used for both components; and, the average length of the COD did not vary systematically from one category to the other when the COD was of moderate duration. 
Other factors did influence the fit of the equation. The one study that used shock avoidance. as the reinforcer (Logue \& de Villiers, 1978) and the two studies that used COSs (Pliskoff, Cicerone, \& Nelson, 1978; Tustin \& Davison, 1979, for the FI $180-$ sec and FI 360 -sec COSs) produced poor fits. The two studies that used two different operanda (Davison \& Ferguson, 1978; McSweeney, 1978) and the two studies that used different reinforcers in the two components (Hollard \& Davison, 1971; Matthews \& Temple, 1979) fell into the intermediate or poor category. All studies that used two different simple schedules also fell into the intermediate or poor category (Herrnstein \& Heyman, 1979; LaBounty \& Reynolds, 1973; Nevin, 1971; Rider, 1979, 1981; Trevett et al., 1972), except Lobb and Davison (1975). The results of Lobb and Davison's study fell into the best category, but were on the borderline. Herrnstein's equation accounted for at least $80 \%$ of the variance in the data on the average for studies in the best category. It accounted for only $80.5 \%$ of the variance in the data for the conc FI VI schedules in the Lobb and Davison study. Therefore, in general, Herrnstein's equation poorly described the results of studies that used different simple schedules as components.

Second, Table 1 supports some conclusions about the size of the $k$ parameter. The obtained values of $\mathbf{k}$ were usually of a size that reasonably represented the asymptotic rates of responding for pigeons pecking keys and pressing treadles, for rats pressing bars, and for people pressing buttons. Eightyfour percent of all values of $k$ fell between 0 and 150 responses/min; and some of the remaining $16 \%$ came from studies in which people pressed buttons, which might be expected to yield higher values of k. Buttonpressing should have a high asymptotic rate of responding because it is a response easy for people to perform quickly.

Tables 1 and 2 also show that $k$ varied systematically with changes in several variables. First, $k$ varied with the species of the subject. The median sizes of $k$ were $61.3,88.0,148.9$, and 5.5 responses/ min for pigeons, rats, people, and cows, respectively. Second, $k$ varied with the simple schedule used in the components. The value of $k$ was usually larger for fixed-ratio (FR) than for VR or mixedratio (MR) components (Rider, 1979), for VR than for VI components (Rider, 1981), and for FI than for FR components (LaBounty \& Reynolds, 1973). The value of $k$ was also larger (median $=143.9$ responses $/ \mathrm{min}$ ) in the one study that used a conc FI FI schedule (White \& Davison, 1973) than it was for all studies combined (median $=65.0$ responses $/ \mathrm{min}$ ). Third, $\mathrm{k}$ varied unsystematically with COD duration but was smaller when a long COS was used. The median sizes of $k$ were $92.7,44.5$, $47.1,42.5,90.0,82.1,74.5$, and 63.8 for $0-, 1-$,
1.5-, 2-, 2.5-, 3-, 5-, and 20-sec CODs, respectively. The median sizes of $k$ were 39.2 and 21.8 for the FI 180 -sec and FI 360 -sec COSs used by Tustin and Davison (1979). Fourth, $k$ was larger when two different simple schedules appeared in the components (median $=129.4$ responses $/ \mathrm{min}$ ) than it was for all studies combined (median $=65.0$ responses $/ \mathrm{min}$ ). Fifth, the median size of $\mathbf{k}$ was smaller for studies that provided different reinforcers in the two components (6.5 responses/min) than for all studies combined (65.0 responses $/ \mathrm{min})$. Finally, $\mathrm{k}$ was smaller for studies that used two different operanda to produce reinforcers in the two components (median $=44.5$ responses $/ \mathrm{min}$ ) than for all studies combined (median $=$ approximately 65.0 responses/ $\min$ ).

Only two variables, the type of concurrent schedule and whether keypecks or treadlepresses produced reinforcers, did not strongly affect the size of $k$. The median size of $k$ was slightly larger for Findley (79.4 responses/min) than for two-operanda (61.3 responses/min) concurrent procedures. But it is unlikely that this represents a significant difference. The value of $k$ was also larger when pigeons pecked keys than when they pressed treadles in one study (McSweeney, 1978) but not in two others (Davison \& Ferguson, 1978; Wheatley \& Engberg, 1978).

Third, Table 1 supports some conclusions about the size of the $\mathbf{R}_{0}$ parameter. In general, the obtained values of $\mathbf{R}_{\mathbf{0}}$ did not correspond well to its interpretation as unprogrammed sources of reinforcement. Only $37 \%$ of all $R_{0}$ parameters fell within the range of 0 to 50 reinforcers/h, the most likely range of values for the present studies. Twenty-three percent of the values of $R_{0}$ were greater than 50 reinforcers $/ h$, which seems too high for the subjects used in these studies (which were deprived of the programmed reinforcers and responded to total rates of programmed reinforcers that rarely exceeded $200 / \mathrm{h}$ ).

Forty percent of all values of $R_{0}$ were also negative. The binomial test could not be applied to the data reported by Bourland and Miller (1981), McSweeney (1975a), McSweeney (1978), Norman and McSweeney (1978), Schmitt (1974), and Trevett et al. (1972, the conc VI VI schedules) because these studies provided fewer than the five points needed for the binomial test to reach significance. Ignoring these studies, 41 of the 70 negative values of $\mathbf{R}_{0}$ reported in Table 1 were significantly less than 0 . Negative values of $R_{0}$ seem to violate Herrnstein's interpretation of this parameter as unprogrammed reinforcers.

Tables 1 and 2 also show that $\mathbf{R}_{\mathbf{0}}$ varied systematically with several variables. First, $\mathbf{R}_{\mathbf{0}}$ varied with the species of subject. The median sizes of $\mathbf{R}_{\mathbf{0}}$ were $7.5,25.1,-9.1$, and -23.9 for pigeons, rats, people, and cows, respectively. Second, $R_{0}$ varied with some 
simple schedules provided by the components. $\mathbf{R}_{\mathbf{0}}$ was larger for FI than for FR components (LaBounty \& Reynolds, 1973). $\mathbf{R}_{0}$ was also larger (median = 19.4 reinforcers $/ h$ ) for the one study that used a conc FI FI schedule (White \& Davison, 1973) than it was for all studies combined (median $=9.5$ reinforcers $/ h$ ). Third, $R_{0}$ was larger for studies that used two different simple schedules in the components (median $=16.5$ reinforcers $/ h$ ) than for all studies combined (median $=9.5$ reinforcers $/ \mathrm{h}$ ). Fourth, $\mathbf{R}_{0}$ was slightly larger for studies using two different operanda in the components (median = 13.3 reinforcers $/ h$ ) than for all studies combined (median $=9.5$ reinforcers $/ \mathrm{h}$ ). Fifth, $R_{0}$ was smaller for studies presenting two different reinforcers in the components $($ median $=-13.9$ reinforcers $/ \mathrm{h}$ ) than it was for all studies combined (median $=9.5$ reinforcers $/ h$ ). In fact, 11 of 13 values of $R_{0}$ were negative when two different reinforcers were used. Finally, $R_{0}$ changed as a U-shaped function of COD duration. The median size of $R_{0}$ was $22.6,7.3,-6.5,-8.0$, $7.4,8.2,19.6$, and 50.0 for $0-, 1-, 1.5-, 2-, 2.5-, 3-$, 5 -, and 20 -sec CODs respectively.

Only three variables did not systematically change the size of $\mathbf{R}_{\mathbf{0}}$. First, $\mathbf{R}_{\mathbf{0}}$ was not systematically different for Findley (median $=9.9$ reinforcers $/ h$ ) and two-operanda (median $=7.3$ reinforcers $/ \mathrm{h}$ ) concurrent procedures. Second, $R_{0}$ did not differ systematically for some differences in component schedules. There were no consistent differences in the size of $\mathbf{R}_{\mathbf{0}}$ between FR and VR or MR components (Rider, 1979), or between FI and VR components (Rider, 1981). Third, $R_{0}$ did not usually vary with the operanda used in the components. $R_{0}$ for keypecking was not systematically different from $R_{0}$ for lever- (Davison \& Ferguson, 1978) or treadlepressing (McSweeney, 1978). $\mathbf{R}_{0}$ was smaller for keypecking than for treadlepressing in Wheatley and Engberg (1978), but this conclusion is suspect because it is based on only two points.

\section{DISCUSSION}

The present data must be treated with caution. Few of these studies were explicitly designed to test Herrnstein's equation. Therefore, procedural problems may prevent some of them from providing an optimal test of the theory. However, the present data will not be misinterpreted if they are taken as providing an overview of how well Herrnstein's theory describes the existing concurrent schedule literature, rather than as an optimal test of this theory. Viewed in this light, several conclusions emerge from the present analysis.

First, Herrnstein's equation described the data moderately well in some situations. It accounted for more than $80 \%$ of the variance in the data for the approximately one-third of the studies that used a moderate-duration COD and that used the same positive reinforcers, operanda, and simple schedules in the two components. This success should not be misinterpreted, however. Duncan and Silberberg (1982) pointed out that goodness-of-fit tests, by themselves, cannot confirm a theory. A theory might produce an adequate fit even if it were in error in several ways. The predictions of the theory must also be confirmed by experiments explicitly designed to test them. As mentioned in the introduction, some of these experiments have been carried out with mixed success. Therefore, the present success implies that Herrnstein's theory is worth further testing in these situations, not that the theory is correct.

Second, Herrnstein's equation did not provide an adequate general description of concurrent schedule responding. It failed to describe the data well in more than two-thirds of the studies, including those that used negative reinforcers, COSs, different reinforcers, different simple schedules, or different operanda in the components. This failure suggests that an alternative theory is needed to describe the results of these studies.

Detailed consideration of alternative theories is beyond the scope of this paper. In some cases, minor modifications might account for the data. For example, Staddon (in press) suggested that responses differ in their sensitivity to reinforcement. Therefore, a revision of Herrnstein's equation that included this variable might account for responding during schedules that use different operanda in the two components. Greater revisions might be required in other cases. For example, Rachlin, Kagel, and Battalio (1980) suggested that different reinforcers may control behavior in different ways. In that case, entirely different theories might have to be formulated for studies using different reinforcers.

Future theories should also look for order in aspects of concurrent-schedule responding other than those addressed by Herrnstein's equation. For example, McSweeney, Melville, Buck, and Whipple (in press) found some order in the local rates of responding. Local rates of responding are the number of responses emitted during a component of a concurrent schedule divided by the time spent responding on that component. Local rates contrast with the overall rates of responding described by Herrnstein's theory. Overall rates are the number of responses emitted during a component divided by total session time.

Local rates might be worth examining because McSweeney et al. (in press) found that local rates often changed as an orderely function of the local rates of reinforcement. Local rates might also be 
worth examining because the assumption that originally led Herrnstein to formulate his theory in terms of overall rates appears, in retrospect, to be incorrect. Herrnstein's theory was originally formulated in terms of overall rates because he assumed that the equation for the absolute rates of responding must yield Equation 1 when their ratio is taken. Equation 1 would follow if the overall rates were used, because the time denominators would be equal for the two components and would cancel when the ratios were taken. Equation 1 would not follow if local rates were used, because the time denominators used to calculate local rates would usually not be equal and would not cancel. But, lately, several authors have questioned whether Equation 1 does describe the data (e.g., Baum, 1974, 1979; Myers \& Myers, 1977; Wearden \& Burgess, 1982). The failure of Equation 1 makes Herrnstein's reason for examining overall rates less compelling and suggests that future experiments should look for order in local rates, as well as in overall rates.

Third, the present data tentatively suggest that changes must be made in Herrnstein's description of his parameters if his equation is to be retained as a description of some studies. Because of their limitations, the present conclusion about $k$ and $\mathbf{R}_{\mathbf{0}}$ must remain tentative. Conclusions based on comparisons of the size of $k$ and $R_{0}$ between the two components of concurrent schedules within single studies are frequently based on the results of only one or two studies. Conclusions based on comparisons of the median size of $k$ and $R_{0}$ across studies are difficult to interpret. For example, if a difference in the median size of a parameter appeared between studies using cows and those using rats, then these results have been summarized by saying that different species respond differently. But, without further experimentation, other procedural differences between the studies, such as the response operanda used, may have actually produced the results. Additionally, no statistical tests confirmed the significance of the differences in the median size of $\mathbf{k}$ and $\mathbf{R}_{\mathbf{0}}$ across studies. Substantial differences in the size of the parameters were required before a variable was said to change the size of that parameter. But, no statistical tests were applied because it was difficult to assess how well their requirements would be met. However, in spite of these limitations, the present data do provide a general picture of the $k$ and $R_{0}$ parameters that violate Herrnstein's interpretation.

The obtained values of the $k$ parameters corresponded to Herrnstein's interpretation in some ways, but not in others. The size of $k$ was generally appropriate to represent the asymptotic rate of responding, and the $\mathbf{k}$ parameter varied in several ways that it should if it is measured in responses per time unit, as Herrnstein argues. For example, $\mathrm{k}$ generally fell between 0 and 150 responses/min, and was sometimes higher for easier responses, such as buttonpressing, as it should be if it is the subject's asymptotic rate of responding. The size of $k$ also varied with the species of the subject and sometimes with the reinforcer provided by the components (Matthews \& Temple, 1979). The size of $k$ should vary with species if it is measured in responses per time unit, because different response operanda are typically used for different species. The value of $\mathbf{k}$ might also vary with the reinforcer, because the form of the response may change with changes in the reinforcer (Jenkins \& Moore, 1973).

However, $k$ also varied with other variables, which seem to violate the assumption that $k$ is constant when measured in constant response units. For example, $\mathrm{k}$ varied with the simple schedule used in the components, and with the changeover requirement. Comparing across studies, $\mathrm{k}$ was also systematically different for studies using two different simple schedules as components than for those using the same ones. This systematic variation in $\mathbf{k}$ questions Herrnstein's interpretation of $k$ as constant (Herrnstein, 1974).

The present data also suggest that Herrnstein's assumption that $\mathbf{R}_{\mathbf{0}}$ represents reinforcers from unprogrammed sources must be modified if his equation is to be retained as a description of some responding. Table 1 showed that the obtained values of $R_{0}$ were frequently negative or too large to fit this description well. The obtained negative $\mathbf{R}_{\mathbf{0}} \mathbf{s}$ create particular problems for Herrnstein's theory. There is no obvious interpretation for negative rates of reinforcement, and the existence of negative $R_{0} S$ may create formal problems for Herrnstein's theory. If the absolute value of a negative $R_{0}$ is greater than the value of $R_{1}+R_{2}$, then Herrnstein's equation predicts negative response rates. If $R_{1}+R_{2}$ is slightly larger than the absolute value of a negative $R_{0}$, then response rates will be close to infinity and will decrease as $\mathbf{R}_{1}+\mathbf{R}_{\mathbf{2}}$ increases. These predictions do not seem reasonable. ${ }^{2}$

The problems created by negative values of $\mathbf{R}_{0}$ might be overcome by forcing $R_{0}$ to be positive and by recalculating the size of $k$ and the proportion of the variance accounted for. This could be done, and, in many cases, Herrnstein's equation would fit almost as well as it does with the present negative values.

However, two problems would remain if $\mathbf{R}_{0}$ were forced to be positive. First, forcing $R_{0}$ to be positive would prevent us from considering alternative theories that are not tied to Herrnstein's interpretation of his parameters (e.g., McDowell, 1980). Second, forcing $R_{0}$ to be positive would still not rescue Herrnstein's interpretation of $R_{0}$. Even if $R_{0}$ was forced to be positive, the variation in $R_{0}$ would still not correspond to that which would be 
expected from unprogrammed reinforcers. $R_{0}$ varied with some factors that intuition suggests should not alter unprogrammed sources of reinforcement. The size of $R_{0}$ was different for FI than for FR schedules, and it varied with the species of subjects and COD duration. $R_{\mathbf{0}}$ was different for concurrent schedules using the same simple schedules in both components, than for those using different simple schedules; it was different for schedules using different operanda in the two components than for those using the same operandum; and it was different for schedules using different reinforcers than for those using the same reinforcers.

Therefore, the present results imply that the interpretation of $\mathbf{R}_{\mathbf{0}}$ as an unprogrammed source of reinforcement should probably be avoided until further studies can determine the size that $\mathbf{R}_{\mathbf{0}}$ typically takes and the variables that produce changes in that size.

The present results do not support or reject Herrnstein's assumption that responding follows the same basic laws during several different schedules. Herrnstein's equation appears to describe simple schedule responding well (de Villiers, 1977; de Villiers \& Herrnstein, 1976). But, the present data and the problems with Herrnstein's equation cited in the introduction show that it does not always describe the existing concurrent schedule literature well. Additionally, several studies show that the modification of Equation 2, which Herrnstein (1970) proposed for multiple schedules, is also incorrect. Contrary to what Herrnstein's theory predicts, Edmon (1978) did not find an increase in the rates of responding as the components of the multiple schedule got longer. Several studies nave also shown that Herrnstein's theory makes incorrect predictions about the relation between simple and multiple-schedule responding (see McSweeney, 1980). As a result, Herrnstein's theory does not provide an adequate description of responding on concurrent and multiple schedules. Only further studies will indicate whether other theories that successfully describe responding on several different schedules can be formulated.

\section{REFERENCES}

BaUM, W. M. On two types of deviation from the matching law: Bias and undermatching. Journal of the Experimental Analysis of Behavior, 1974, 22, 231-242.

BAUM, W. M. Matching, undermatching, and overmatching in studies of choice. Journal of the Experimental Analysis of Behavior, 1979, 32, 269-281.

Bourland, G., \& Miller, J. T. The role of discriminative stimuli in concurrent performances. Journal of the Experimental Analysis of Behavior, 1981, 36, 231-239.

Bradshaw, C. M., Szabadi, E., \& Bevan, P. Relationship between response rate and reinforcement frequency in variableinterval schedules: The effect of the concentration of sucrose reinforcement. Journal of the Experimental Analysis of Behavior, 1978, 29, 447-452.

Bradshaw, C. M., Szabadi, E., Bevan, P., \& Ruddle, H. V. The effect of signaled reinforcement availability on concurrent performances in humans. Journal of the Experimental Analysis of Behavior, 1979, 32, 65-74.

Catania, A. C. Concurrent performances: Reinforcement interaction and response independence. Journal of the Experimental Analysis of Behavior, 1963, 6, 253-263.

Catania, A. C., \& REYnolds, G. S. A quantitative analysis of the responding maintained by interval schedules of reinforcement. Journal of the Experimental Analysis of Behavior, 1968, 11, 327-383.

Davison, M. Preference in concurrent variable-interval fixed-ratio schedules. Journal of the Experimental Analysis of Behavior, $1982,37,81-96$.

Davison, M., \& Ferguson, A. The effects of different component response requirements in multiple and concurrent schedules. Journal of the Experimental Analysis of Behavior, 1978, 29, 283-295.

Davison, M. C., \& Hunter, I. W. Performance on variableinterval schedules arranged singly or concurrently. Journal of the Experimental Analysis of Behavior, 1976, 25, 335-345.

DE VILlie Rs, P. A. Choice in concurrent schedules and a quantitative formulation of the law of effect. In W. K. Honig \& J. E. R. Staddon (Eds.), Handbook of operant behavior. Englewood Cliffs, N.J: Prentice-Hall, 1977.

de Villiers, P. A., \& Herrnstein, R. J. Toward a law of response strength. Psychological Bulletin, 1976, 83, 1131-1153.

Duncan, H. J., \& Sruberbera, A. The effects of concurrent responding and reinforcement on behavioral output. Journal of the Experimental Analysis of Behavior, 1982, 38, 125-132.

Edmon, E. L. Multiple schedule component duration: A reanalysis of Shimp and Wheatley (1971) and Todorov (1972). Journal of the Experimental Analysis of Behavior, 1978, 30, 239-241.

Fantino, E., Squires, N., Delarück, N., \& Peterson, C. Choice behavior and the accessibility of the reinforcer. Journal of the Experimental Analysis of Behavior, 1972, 18, 35-43.

FARLEY, J. Reinforcement and punishment effects in concurrent schedules: A test of two models. Journal of the Experimental Analysis of Behavior, 1980, 33, 311-326.

Findley, J. D. Preference and switching under concurrent scheduling. Journal of the Experimental Analysis of Behavior, 1958, 1, 123-144.

Fleshler, M., \& Hofrman, H. S. A progression for generating variable-interval schedules. Journal of the Experimental Analysis of Behavior, 1962, 5, 529-530.

Graft, D. A., Len, S. E. G., \& Whitworth, T. L. The matching law in and within groups of rats. Journal of the Experimental A nalysis of Behavior, 1977, 27, 183-194.

Herrnste in, R. J. Relative and absolute strength of response as a function of frequency of reinforcement. Journal of the Experimental Analysis of Behavior, 1961, 4, 267-272.

Herrnste in, R. J. On the law of effect. Journal of the Experimental Analysis of Behavior, 1970, 13, 243-266.

Herratein, R. J. Formal properties of the matching law. Journal of the Experimental Analysis of Behavior, 1974, 21, 159-164.

Herrngte in, R. J., \& HeYMan, G. M. Is matching compatible with reinforcement maximization on concurrent variable interval variable ratio? Journal of the Experimental Analysis of Behavior, 1979, 31, 209-223.

Herrate in, R. J., \& Loveland, D. H. Hunger and contrast in a multiple schedule. Journal of the Experimental Analysis of Behavior, 1974, 21, 511-517.

Hollard, V., \& Davison, M. C. Preference for qualitatively different reinforcers. Journal of the Experimental Analysis of Behavior, 1971, 16, 375-380.

Jenkins, H. M.. \& Moore, B. R. The form of the auto-shaped response with food or water reinforcers. Journal of the Experimental Analysis of Behavior, 1973, 20, 163-181. 
LABounty, C. E., \& ReYnolds, G. S. An analysis of response and time matching to reinforcement in concurrent ratio-interval schedules. Journal of the Experimental Analysis of Behavior, $1973,19,155-166$.

Leigland, S. M. Deviations from matching as a measure of preference for alternatives in pigeons. Journal of the Experimental Analysis of Behavior, 1979, 32, 1-13.

Loвb, B., \& DAvison, M. C. Performance in concurrent interval schedules: A systematic replication. Journal of the Experimental Analysis of Behavior, 1975, 24, 191-197.

Logue, A. W., \& de Villiens, P. A. Matching in concurrent variable-interval avoidances schedules. Journal of the Experimental Analysis of Behavior, 1978, 29, 61-66.

Matthews, L. R., \& Temple, W. Concurrent schedule assessment of food preferences in cows. Journal of the Experimental Analysis of Behavior, 1979, 32, 245-254.

MCDowell, J. J. An analytic comparison of Herrnstein's equations and a multivariate rate equation. Journal of the Experimental Analysis of Behavior, 1980, 33, 397-408.

McDowelL., J. J. Wilkinson's method of estimating the parameters of Herrnstein's hyperbola. Journal of the Experimental Analysis of Behavior, 1981, 35, 413-414.

MCSWEeney, F. K. Concurrent schedule responding as a function of body weight. Animal Learning \& Behavior, 1975, 3, 264-270. (a)

McSweEnEy, F. K. Matching and contrast on several concurrent treadle-press schedules. Journal of the Experimental Analysis of Behavior, 1975, 23, 193-198. (b)

MCSwEeney, F. K. Sum of responding as a function of sum of reinforcement on two-key concurrent schedules. Animal Learning \& Behavior, 1977, 5, 110-114.

McSweeney, F. K. Prediction of concurrent keypeck treadlepress responding from simple schedule performance. Animal Learning \& Behavior, 1978, 6, 444-450.

MCSWEENEY, F. K. Differences between rates of responding emitted during simple and multiple schedules. Animal Learning \& Behavior, 1980, 8, 392-400.

McSweeney, F. K. Prediction of concurrent treadle-pressing from simple schedule performance. Behaviour A nalysis Letters, $1982,2,11-20$.

MCSweeneY, F. K., \& Dericco, D. A. Rates of responding in the pigeon generated by simple and complex schedules which provide the same rates of reinforcement. Animal Learning \& Behavior, 1976, 4, 379-385.

McSweeney, F. K., Melville, C. L., Buck, M. A., \& Whipple, J. E. Local rates of responding and reinforcement during concurrent schedules. Journal of the Experimental Analysis of Behavior, in press.

MYERs, D. L., \& MYERS, L. E. Undermatching: A reappraisal of performance on concurrent variable-interval schedules of reinforcement. Journal of the Experimental Analysis of Behavior, 1977, 25, 203-214.

Nevin, J. A. Rates and patterns of responding with concurrent fixed interval and variable interval reinforcement. Journal of the Experimental Analysis of Behavior, 1971, 16, 241-247.

Norman, W. D., \& MCSweeney, F. K. Matching, contrast and equalizing in the concurrent lever press responding of rats. Journal of the Experimental Analysis of Behavior, 1978, 29, 453-462.

Pliskoff, S. S., Cicerone, R., \& Nelson, T. D. Local responserate constancy on concurrent variable-interval schedules of reinforcement. Journal of the Experimental Analysis of Behavior, 1978, 29, 431-446.
Rachlin, H. C. Contrast and matching. Psychological Review, $1973,80,217-234$.

RAchlin, H., \& Baum, W. M. Effects of alternative reinforcement: Does the source matter? Journal of the Experimental Analysis of Behavior, 1972, 18, 231-241.

Rachlin, H., Kagel, J. H., \& Battalio, R. C. Substitutability in time allocation. Psychological Review, 1980, 87, 355-374.

RIDE R, D. P. Concurrent ratio schedules: Fixed vs. variable response requirements. Journal of the Experimental Analysis of Behavior, 1979, 31, 225-237.

RIDER, D. P. Concurrent fixed-interval variable-ratio schedules and the matching relation. Journal of the Experimental Analysis of Behavior, 1981, 36, 317-328

SchмiтT, D. R. Effects of reinforcement rate and reinforcer magnitude on choice behavior of humans. Journal of the Experimental Analysis of Behavior, 1974, 21, 409-419.

Segal, E. F. A rapid procedure for generating random reinforcement intervals on VI and VR tapes. Journal of the Experimental Analysis of Behavior, 1964, 7, 20.

Siegel, S. Nonparametric statistics for the behavioral sciences. New York: McGraw-Hill, 1956.

Staddon, J. E. R. Behavioral competition, contrast and matching. In M. Commons, R. Herrnstein, \& H. Rachlin (Eds.), Quantitative analyses of behavior: Matching and maximizing accounts. Cambridge, Mass: Ballinger Press, in press.

Stubss, D. A., \& Pliskofr, S. S. Concurrent responding with fixed relative rate of reinforcement. Journal of the Experimental Analysis of Behavior, 1969, 12, 887-895.

Trevet, A. J., Davison, M. C., \& Williams, R. J. Performance in concurrent interval schedules. Journal of the Experimental A nalysis of Behavior, 1972, 17, 369-374.

Tustin, R. D., \& DAvison, M. Choice: Effects of changeover schedule on concurrent performance. Journal of the Experimental Analysis of Behavior, 1979, 32, 75-91.

WeArden, J. H., \& Burgess, I. S. Matching since Baum (1979). Journal of the Experimental Analysis of Behavior, 1982, 38, 339-348.

Wetherington, C. L., \& Lucas, T. R. A note on fitting Herrnstein's equation. Journal of the Experimental Analysis of Behavior, 1980, 34, 199-206.

Whe AtLey, K. L., \& Enaberg, L. A. Choice performance in several concurrent key-peck treadle-press reinforcement schedules. Journal of the Experimental Analysis of Behavior, 1978, 29, 181-190.

White, A. J., \& Davison, M. C. Performance in concurrent fixed-interval schedules. Journal of the Experimental Analysis of Behavior, 1973, 19, 147-153.

\section{NOTES}

1. There appears to be an error in Equation 8 in Wetherington and Lucas (1980). Equation 8 should be

$$
k=\sum \frac{\mathbf{R}_{i} r_{i}}{\left(r_{i}+r_{e}\right)} \div \sum \frac{r_{i}^{2}}{\left(r_{i}+r_{e}\right)^{2}}
$$

2. The authors are indebted to Michael Davison for pointing out the problems created by negative values of $\mathbf{R}_{\mathbf{0}}$.

(Manuscript received September 30, 1982; revision accepted for publication February 15, 1983.) 